\title{
DEEP LEARNING BASED ROAD RECOGNITION FOR INTELLIGENT SUSPENSION SYSTEMS
}

\author{
JINWEI SUN \\ School of Vehicle Engineering, Xi'an Aeronautical University, Xi'an, China \\ JiNGYU CONG \\ School of Information and Communication Engineering, Hainan University, Haikou, China \\ e-mail: jingyuconghnu@126.com
}

\begin{abstract}
This paper presents a deep learning-based road recognition strategy for advanced suspension systems. A four-quarter suspension model with a magnetorheological (MR) damper is developed, and four typical road images with corresponding roughness data are collected. A back-propagation neural network based autoencoder and Convolutional Neural Networks $(\mathrm{CNN})$ are utilized to form the deep learning structure. By utilizing the multi-object genetic algorithm, the optimal parameters can be obtained, and the control current can be adaptively adjusted. Simulation results indicate that the designed structure can identify the road type accurately, and the recognition-based control strategy can improve the suspension performance effectively.
\end{abstract}

Keywords: intelligent suspension system, deep learning, road recognition

\section{Introduction}

The latest developments of vehicle engineering have brought expanding business request to enchain passengers' comfort, and the current broadly used suspension systems can hardly make attained without sacrificing handling stability (Qin et al., 2018). To solve this, active and semi-active suspension frameworks need to be developed as a feasible option and have been broadly studied in the recent years for their better trade-off between comfort and stability (Qin et al., 2018; Krzyżyński and Maciejewski, 2019). Morales et al. (2016) designed an active suspension control system with adjustable damping and stiffness by using a magnetorheological (MR) shock absorber and pneumatic system, which achieve the same roll angle levels as a similar passive system while reducing sprung mass acceleration by up to $30 \%$. A semi-active seat suspension model with an MR damper was presented by Maciejewski et al. (2019) to reduce the horizontal direction vibration. An actuator motion based semi-active variable damping control method was proposed by Gong and Chen (2020) to improve the vertical performance of heavy vehicles.

Active and semi-active control can effectively reduce the impact of vibration on the human body, and most of current researches take the road input as a bounded external disturbance to design control parameters (Sun et al., 2019). In real applications, road conditions are varying significantly and should be considered in the controller design process. That means to design appropriate control parameters for different road conditions. With respect to the specific issue, many adaptive control strategies with adjustable parameters according to various road conditions have been proposed (Liu et al., 2020; Qin et al., 2019). Under the circumstance, road recognition is the sticking point for practical applications of controllable suspension systems.

In general, the following three methods are used for road recognition: (1) road elevation measurement is carried out by keeping the independently operating road roughness meter in contact with the ground (Doumiati et al., 2011); (2) by installing acceleration or displacement 
sensors on the car, road unevenness is identified through the vehicle dynamic responses, where the recognition results are always statistical characteristics (Qin et al., 2014); (3) use non-contact measurement methods to measure road elevation or identify road types through onboard sensors such as a lidar and camera (Zhang et al., 2015). The first method has high accuracy, but requires special instruments and a high real-time measurement cost, which restrict the engineering application. Owing to the rapid development of intelligent vehicles, the latter two methods have got a wide range of applications by the agency of dynamic sensors, lidar or cameras.

Through the adoption of dynamic control sensors installed in cars, the system response-based method is utilized for road recognition. Qin et al. (2017) provided a deep neural network-based classification method with the input signal of the unsprung mass response, which could obtain relative high accuracy. Apart from the data driven modus, the model-based road estimation strategy has been addressed through the use of the observer technique. To study the fatigue loads of vehicles arising form road roughness, Fauriat et al. (2016) presented an augmented Kalman observer to realize the inverse estimation of road information, and the accuracy factors were also analyzed. Considering the vertical and longitudinal dynamics of vehicles, Rath et al. (2015) combined two different kinds of observers to estimate road roughness and tire road friction simultaneously. All the recent literature with respect to the system response suffer from common weakness, such as accurate extrapolation and unmeasured responses ( $\mathrm{Li}$ et al., 2016). Moreover, the model-based road recognition method mainly concentrates on standard road excitation, and it can hardly be applied to non-road pavements. Lidar or camera based non-contact measurement methods thus arise as an alternative. Viikari et al. (2009) used a $24 \mathrm{GHz}$ millimeter-wave radar to identify road conditions and detect low-friction roads caused by water, ice, or road surface snow. Peterson et al. (2008) adopted a random state estimation instrument to process the road surface data collected from $2 \mathrm{D}$ and $3 \mathrm{D}$ radars and identify the most road surface shape.

Since the high cost of radars hinders its application in mass vehicles, this paper presents a camera-based road recognition framework for controllable suspension systems. One of the major problems with the use of cameras for road recognition is of its scope of application, such as the impact of special circumstances including strong light, weak light, image tilt, and partial lack of images in the natural environment (Bekhti and Kobayashi, 2015). Besides, the traditional backpropagation neural network requires complex preprocessing, original images cannot be directly used as the input of the network. Meanwhile, when there are more neurons in the network, the weight parameters of the network will be very huge, which makes the classification efficiency greatly reduced or even fail to work normally. To deal with these problems and improve the classification efficiency, a deep learning-based road recognition method is designed to identify non-standard pavements for controllable suspension systems, which consists of an autoencoder, a feed forward neural network for road classification, and an adaptive adjustment mechanism of the controllable suspension according to recognition results. An unsupervised back-propagation neural network based autoencoder can learn the hidden features of input images, and the learned features can be used to reconstruct the image data, thus reducing the computational burden of the subsequent network (Hinton and Zemel, 1994). The feedforward neural network consists of a multi-layer Convolutional Neural Networks (CNN) network. Its main advantage is that it can extract features from the input image through simple convolution and pooling operations, and automatically updates network parameters according to the back-propagation algorithm. It is worth noting that the actual suspension system is a complex nonlinear system and different road inputs have a very strong influence on the suspension performance. Therefore, to achieve better control performance of the system, an accurate system model and road recognition algorithm can be used to adjust the control parameters adaptively according to the road type. The main contributions of this work can be summarized as follows: 
- An autoencoder based recognition framework is proposed, which can greatly reduce the amount of training data and improve the training efficiency.

- The CNN based recognition method can accurately recognize the road surface type using only the on-board camera via the deep learning structure.

The rest of study is organized as follows: Section 2 presents the four-quarter controllable suspension model. Network architecture including the autoencoder network and the CNN structure are given in Section 3. Network training and controllable suspension simulation are shown and discussed in Section 4, and Section 5 concludes the study.

\section{Controllable suspension model}

The vehicle suspension system is a type of complex mechanical system with nonlinearity, time-varying, structural and non-structural uncertainties. System nonlinearity and parameter uncertainties are widely present in the suspension system, including structural layout differences, internal damping components, time-varying parameters and structural uncertainty caused by load changes. The system uncertainties mentioned above will cause a resonance frequency shift, time delay and other phenomena, and then cause unknown changes in the dynamic response. For such a complex mechanical system, the more system characteristics that are considered when modeling, the more comprehensive the model can be built, the closer the model is to the actual situation, but at the same time the greater the difficulty of modeling and the higher the difficulty of analysis. On the other hand, the higher the simplification of the established model, the easier the analysis, but the results obtained are often not accurate enough. Therefore, the establishment principle of the vehicle model can be described as: according to the needs of analysis, find a feasible balance between the accuracy and complexity. Furthermore, in the process of vehicle system modeling, the following assumptions are often followed to simplify the system: (1) assume that the load remains constant during the operation of the vehicle model; (2) the tire stiffness is a constant value and the wheel damping is ignored; (3) there is no "wheel jumping" phenomenon, and the wheels always keep in contact with the ground.

\subsection{Suspension model}

The MacPherson suspension system is widely used in vehicle front suspension systems due to its simple structure, small wheel positioning changes, good handling stability and a large engine layout space. The typical MacPherson suspension system is shown in Fig. 1a. It consists of an articulated strut and an A-shaped lower control arm. The shock absorber strut doubles as a kingpin, and the knuckle rotates around this kingpin. The sliding column is hinged to the body through the upper mounting assembly, the inner side of the lower control swing arm is connected to the body through a rubber (hydraulic) bushing; the outer side is hinged to the steering head through a ball joint. Considering that the mass of the lower control arm is much smaller than the sprung and the unsprung mass, ignoring the lower control arm rotation angle $\theta_{1}$ and sliding column rotation angle $\theta_{2}$, the system degenerates to a two degree of freedom model, as shown in Fig. 1b.

The simplified suspension model consists of the sprung mass $m_{b}$, linear spring, MR shock absorber, unsprung mass $m_{s}$ and linear stiffness tire, and the system input $x_{r}$ is the road roughness excitation. The root mean square (RMS) values of the sprung mass acceleration, suspension deflection and tire deflection were considered as the evaluation index of vehicle riding comfort and handling stability. The purpose of the controllable suspension system is to minimize the sprung mass motion and tire deflection while satisfying the suspension deflection constraint. 
(a)

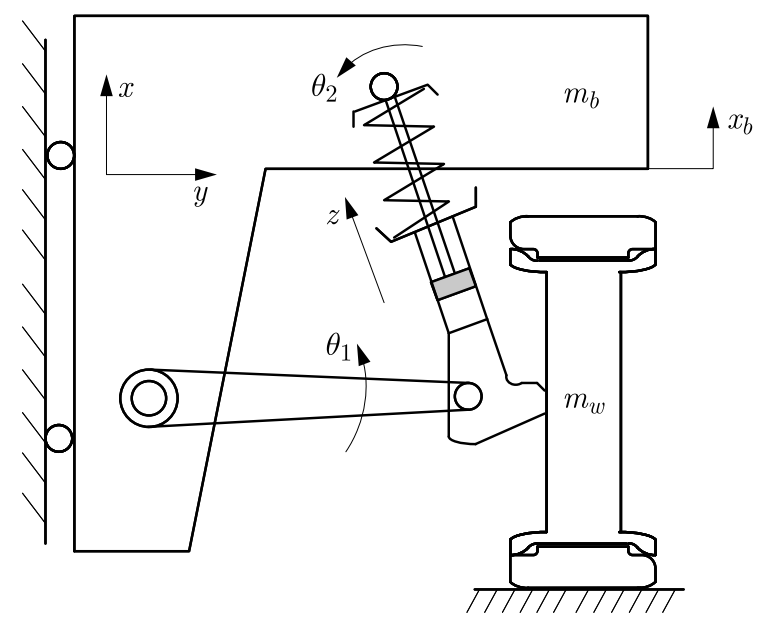

(b)

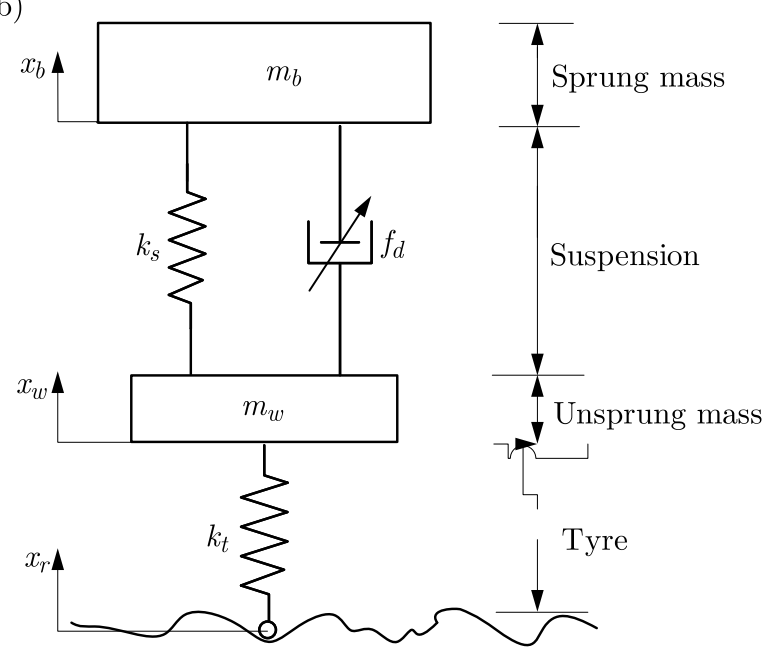

Fig. 1. (a) Typical MacPherson suspension system; (b) simplified suspension model

The semi-active suspension model is as follows:

$$
\begin{aligned}
& m_{b} \ddot{x}_{b}+f_{d}+k_{s}\left(x_{b}-x_{w}\right)=0 \\
& m_{w} \ddot{x}_{w}-f_{d}-k_{s}\left(x_{b}-x_{w}\right)+k_{t}\left(x_{w}-x_{r}\right)=0
\end{aligned}
$$

\subsection{MR shock absorber model}

The external characteristics of MR shock absorbers are usually strongly nonlinear and have hysteresis loops. The accuracy of the MR model has a significant impact on the suspension control. A non-parametric model, pseudo-static model and parametric dynamic model are three commonly used models of MR shock absorbers. Since the parametric dynamic model describes the external characteristics of the MR shock absorber most comprehensively and accurately, the parametric dynamic model proposed by Kwok et al. (2006) is adopted in this paper.

The MR damper model is composed of a hysteresis component, an elastic component and a damping component in parallel. Considering the dynamic characteristics of the MR damper, the mathematical model can be obtained as

$$
f_{d}=c \dot{x}+k x+\alpha z+f_{0} \quad z=\tanh (\beta \dot{x}+\delta \operatorname{sgn}(x))
$$

where $f_{d}, c$ and $k$ are the output damping force, viscous damping coefficient and stiffness coefficient of the shock absorber, respectively. $\beta$ and $\delta$ are the adjustment parameters related to the height of the hysteresis displacement loop. $\alpha, z$ and $f_{0}$ represent the hysteresis factor, hysteresis displacement and damper frictional resistance, respectively. $x$ and $\dot{x}$ are the relative displacement and velocity at both ends of the shock absorber. The experimental data is obtained by means of the test equipment shown in Fig. 2, which can provide insight into MR damper characteristics. The MR damper used in the work is a RD-8041-1 prototype manufactured by the LORD Corporation, which accepts the maximum input current of $2 \mathrm{~A}$ and $12 \mathrm{~V} \mathrm{dc}$. A sinusoidal excitation of a small magnitude $(20 \mathrm{~mm})$ and different frequencies are applied from the damping test system as the displacement input. With the application of a set of magnetizing currents (from $0 \mathrm{~A}$ to $2 \mathrm{~A}$ ), the damping force is measured, and the MR damper characteristics are plotted in Fig. 3. The mathematical expressions of the above undetermined parameters can be obtained through parameter identification of the MR shock absorber, where 


$$
\begin{aligned}
& c=1929 i+1232 \quad k=-1700 i+5100 \\
& \alpha=-244 i^{2}+918 i+32 \quad \beta=100 \\
& \delta=0.3 i+0.58 \quad f_{0}=-18 i-257
\end{aligned}
$$

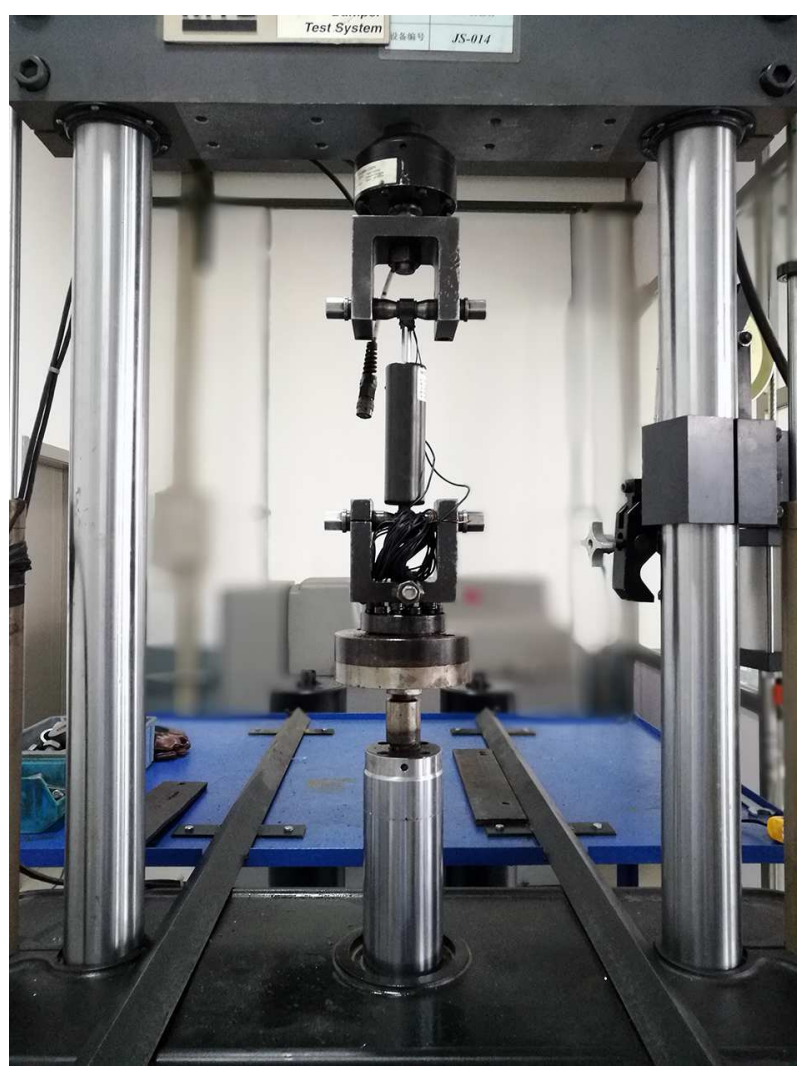

Fig. 2. MR damper test platform
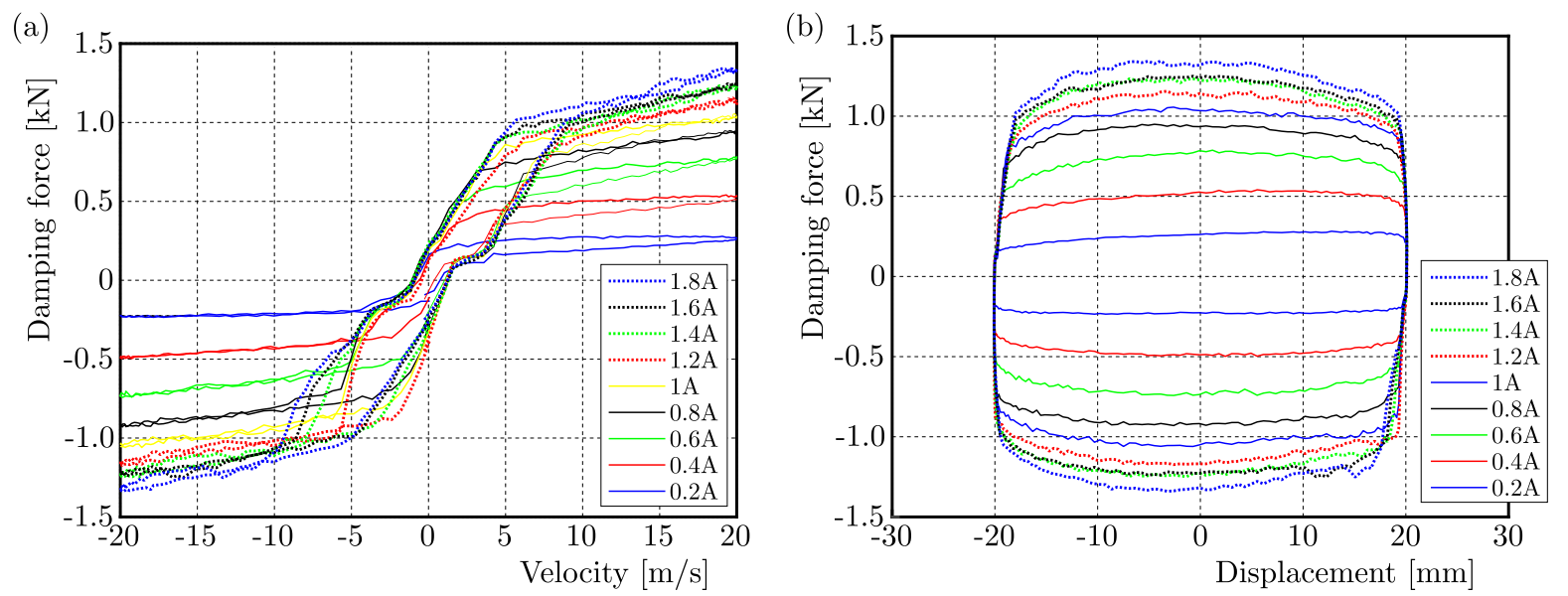

Fig. 3. MR damper characteristics: (a) force-velocity characteristic, (b) force-displacement characteristic

\subsection{Road roughness collection}

The vertical height of the road surface relative to the reference plane is denoted as $q$, and the length along the longitudinal direction of the road is denoted as $I$. The function of $q$ varying with $I$ is called the road surface roughness function, denoted as $q(I)$. The current measurement techniques mainly include fixed reference measurement, follow-up reference measurement, 
dynamic response measurement and an angle reference measurement method. The roughness of asphalt road, cement road, gravel road and pebble road were measured using the angular reference measurement method, and the measured images of the road are shown in Fig. 4a. The roughness curves corresponding to the four types of road surfaces are plotted in Fig. 4b.

(a)

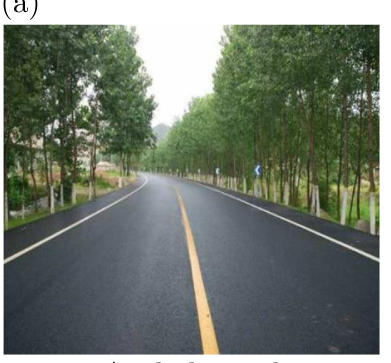

Asphalt road

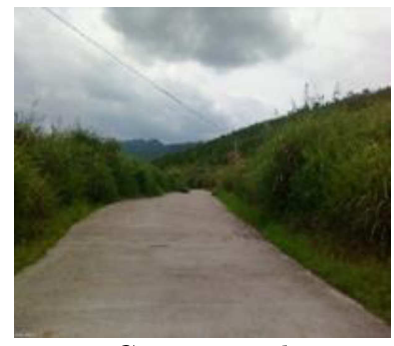

Cement road

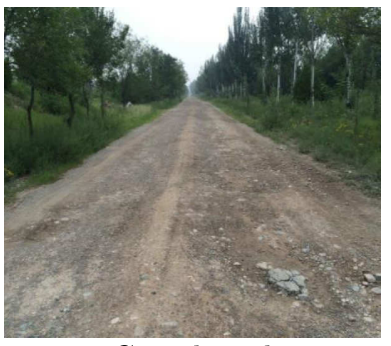

Gravel road

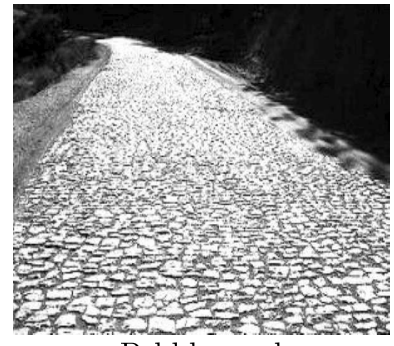

Pebble road

(b)
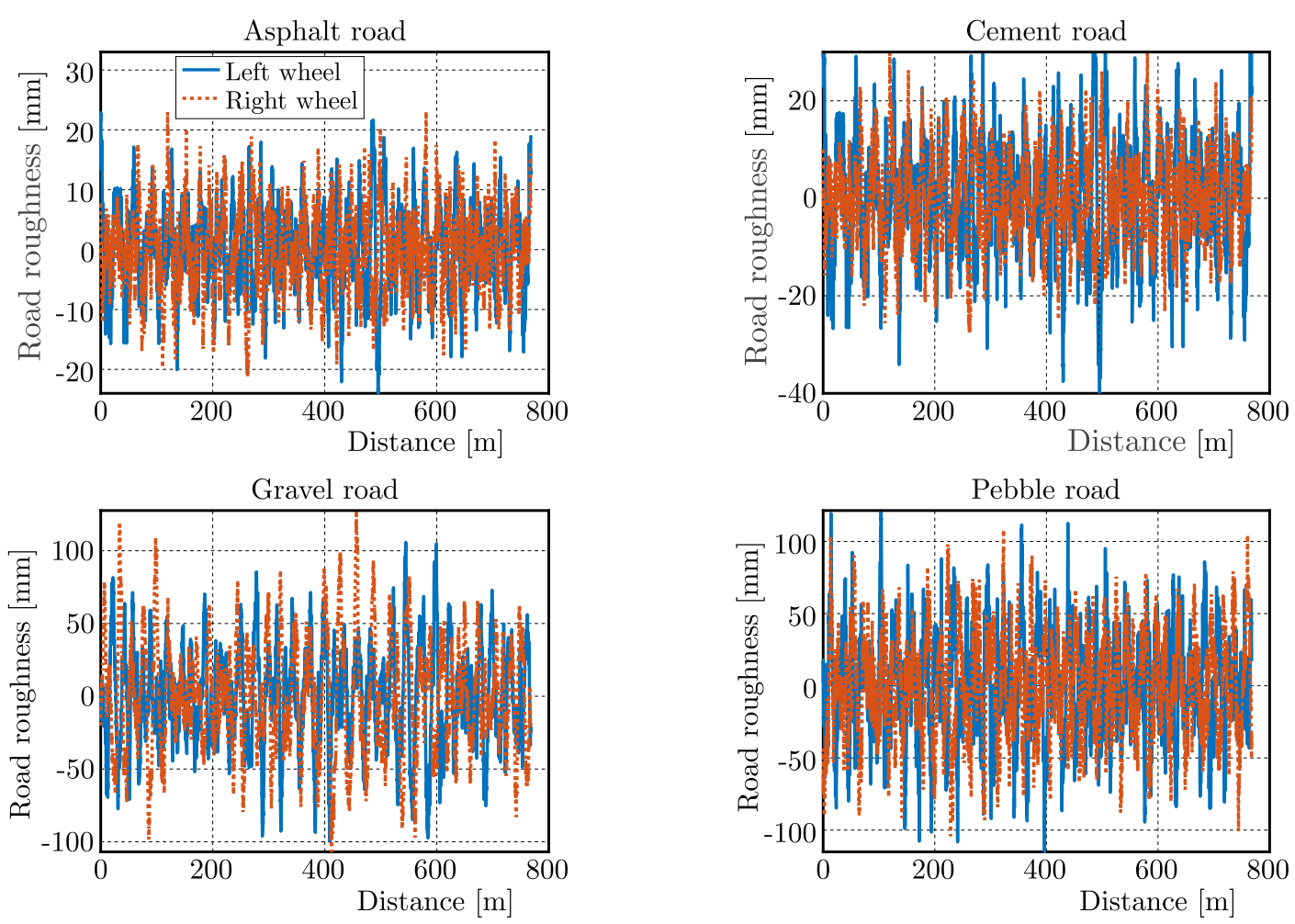

Fig. 4. Road roughness information: (a) road image type, (b) road roughness measurement cureve

\section{Network architecture}

In this Section, the CNN based deep learning method is designed for road image recognition. For the large amount of road pictures, to reduce the data size and improve the learning efficiency of the network, an unsupervised neural network based autoencoder is utilized to preprocess the road images.

\subsection{Autoencoder network}

The autoencoder network is a "large to small, then small to large" structure that compresses the input images to a lower dimension, extracts the main components of the original images, and then restores the main components to the original dimension through a decoder, as shown 
in Fig. 5. A multilayer feedforward neural net is included in the autoencoder, which is trained unsupervised by the decoder to reconstruct the optimization conditions for the approximation between the features and the original features. This process can reduce the size of data and computation burden of the subsequent network while preserving the main features of road images.

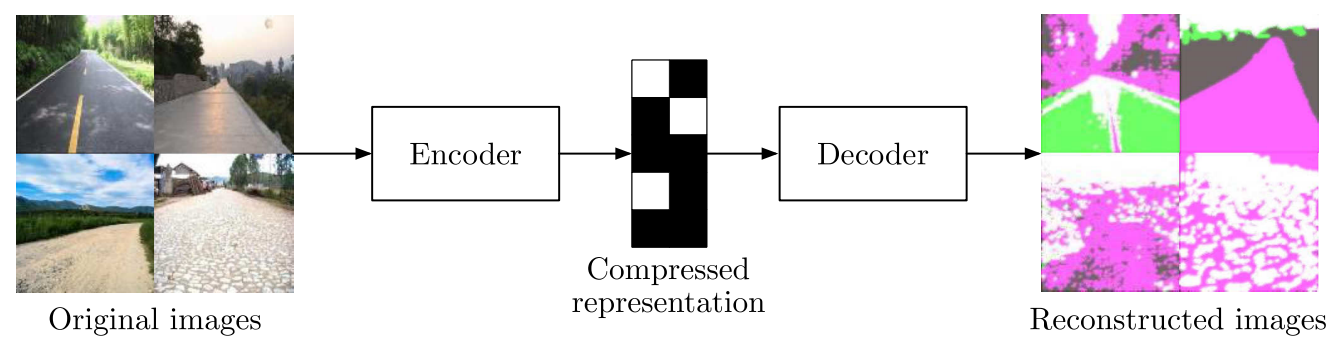

Fig. 5. Autoencoder structure

The input data can be represented by encoding functions

$$
h=f(x):=s_{f}(\mathbf{W} \mathbf{x}+\mathbf{p})
$$

where $s_{f}$ is the activation function of the encoder, usually taken as the sigmoid function

$$
s_{f}(z)=\frac{1}{1+\mathrm{e}^{-z}}
$$

where $W$ and $p$ are the neural network parameters, which denote the weights and biases of the model, respectively. The decode function used in the decode process can be expressed as

$$
r=g(h):=s_{g}\left(\mathbf{W}^{\mathrm{T}} \mathbf{h}+\mathbf{q}\right)
$$

which can map the coding $h$ to the original dimensional space, thus achieving the reproduction of the input data. $s_{g}$ is the activation function of the decoder, which is also taken as the sigmoid function. In order to fit the training samples, the difference between the network output and the corresponding label values needs to be minimized. Based on this, the following loss function model can be given

$$
J(\mathbf{W}, \mathbf{b})=\frac{1}{m} \sum_{i=1}^{m} \frac{1}{2}\left\|h_{w, b}\left(\mathbf{x}^{(i)}\right)-\mathbf{x}^{(i)}\right\|_{2}^{2}+\frac{\lambda}{2}\|\mathbf{W}\|_{2}^{2}
$$

where $\|\bullet\|_{2}$ denotes the $L_{2}$ norm, $\mathbf{x}^{(i)}$ represents the $i$-th sample, and $\lambda$ is the hyper parameter. The first term in the formula is to fit the training sample while the second term can reduce the amplitude of the weight and prevent overfitting.

If the output is approximately equal to the input data, the reconstruction error can be minimized by adjusting the parameters of the encoding and decoding stages.

\section{2. $\quad$ CNN structure}

In general, CNN contains the input layer, convolutional layer, pooling layer and fully connected layer. The convolutional layer comes with an activation function as the Rectified Linear Unit (ReLU), and the combination of convolutional layer and pooling layer can appear multiple times in the hidden layer. The fully connected layer is located after the convolutional layer and connected to the Softmax output layer. In this paper, we build the corresponding CNN to learn different road image data, and finally realize the purpose of road recognition. The constructed CNN structure is shown in Fig. 6, including 13 convolutional layers, 5 pooling layers, 3 fully 
connected layers, and finally the Softmax output layer. The parameters of the CNN are listed in Table 1. For the image data, the operation of the convolution layer is as follows

$$
\begin{aligned}
& Z^{l+1}(i, j)=\left[Z^{l} \otimes w^{l}\right](i, j)+b=\sum_{k=1}^{k_{l}} \sum_{x=1}^{f} \sum_{y=1}^{f}\left[Z_{k}^{l}\left(s_{0} i+x, s_{0} j+y\right) w_{k}^{l+1}(x, y)\right]+b \\
& (i, j) \in\left\{0,1, \ldots, L_{l+1}\right\} \quad L_{l+1}=\frac{L_{l}+2 p-f}{s_{0}}+1
\end{aligned}
$$

where $Z^{l}, Z^{l+1}$ are the input and output of $l+1$ convolution layer respectively, also known as feature maps. $w^{l}$ is the convolution kernel, $b$ is the bias value, and $L_{l+1}$ represents the size of $Z^{l+1}$. It is assumed that the feature maps have the same length and width. $Z^{l+1}(i, j)$ denotes the pixel value of a point in the feature map, and $K$ is the number of channels in the feature maps: $K=1$ if the input is a grayscale map, and $K=3$ if it is a color map. $s_{0}, p$ and $f$ are the step size of the convolution, the number of filling layers and the size of the convolution kernel, respectively.

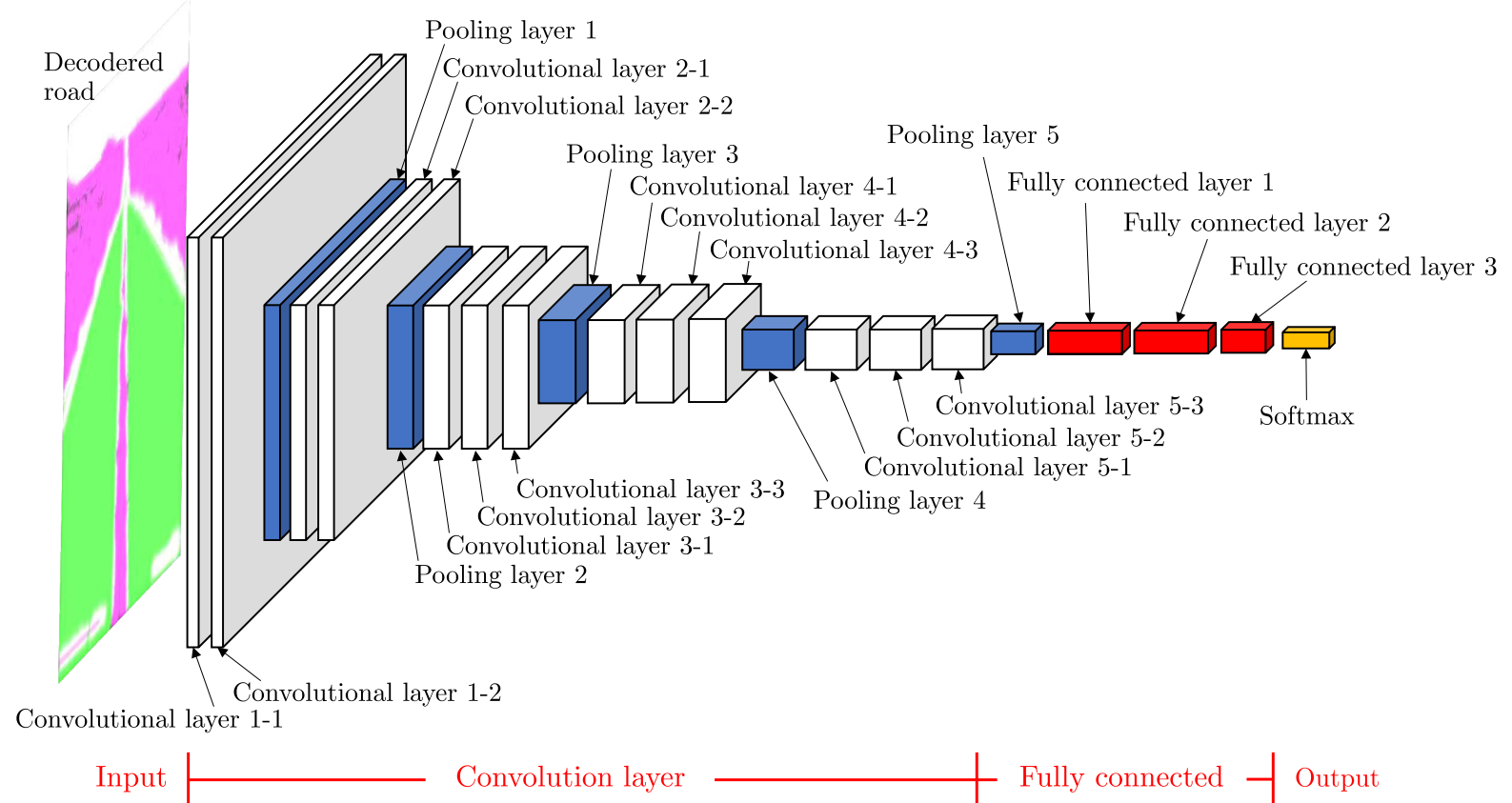

Fig. 6. CNN structure

After feature extraction in the convolution layer, the output feature map is passed to the pooling layer for feature selection, where the pixel values of individual points in the feature map are replaced with the statistics of the pixel values in their adjacent regions for the purpose of dimensionality reduction. Maximum pooling is utilized in this paper, where the extreme pixel value in the adjacent region is selected.

When the convolution kernel is of size $f=1$, step size $s_{0}=1$ and does not contain padding, the equation represents the matrix multiplication operation within the fully connected layer

$$
Z^{l+1}=\sum_{k=1}^{k_{l}} \sum_{i=1}^{L} \sum_{j=1}^{L}\left(Z_{i, j, k}^{l} w_{k}^{l+1}\right)+b
$$

where $Z_{i, j, k}^{l}$ is the pixel value of the fully connected layer input feature map in the $k$ channel at the point $(i, j) ; w_{k}^{l+1}$ is the weight value of the convolution kernel in the $k$ channel. 
Table 1. CNN parameters

\begin{tabular}{|l|c|c||l|c|c|}
\hline \multicolumn{1}{|c|}{ Type } & Kernel size & No. & Type & Kernel size & No. \\
\hline \hline Batch size & & 128 & Convolution layer 4-1 & $3 \times 3 \times 256$ & 512 \\
\hline Convolution layer 1-1 & $3 \times 3 \times 3$ & 64 & Convolution layer 4-2 & $3 \times 3 \times 512$ & 512 \\
\hline Convolution layer 1-2 & $3 \times 3 \times 64$ & 64 & Convolution layer 4-3 & $3 \times 3 \times 512$ & 512 \\
\hline Pooling layer 1 & $2 \times 2$ & & Pooling layer 4 & $2 \times 2$ & \\
\hline Convolution layer 2-1 & $3 \times 3 \times 64$ & 128 & Convolution layer 5-1 & $3 \times 3 \times 512$ & 512 \\
\hline Convolution layer 2-2 & $3 \times 3 \times 128$ & 128 & Convolution layer 5-2 & $3 \times 3 \times 512$ & 512 \\
\hline Pooling layer 2 & $2 \times 2$ & & Convolution layer 5-3 & $3 \times 3 \times 512$ & 512 \\
\hline Convolution layer 3-1 & $3 \times 3 \times 128$ & 256 & Pooling layer 5 & $2 \times 2$ & \\
\hline Convolution layer 3-2 & $3 \times 3 \times 256$ & 256 & Fully connected layer 1 & & 4096 \\
\hline Convolution layer 3-3 & $3 \times 3 \times 256$ & 256 & Fully connected layer 2 & & 4096 \\
\hline Pooling layer 3 & $2 \times 2$ & & Fully connected layer 3 & & 4 \\
\hline
\end{tabular}

The Softmax function can compress an $N$-dimensional vector $\mathbf{p}$ which containing arbitrary real numbers into another $N$-dimensional real vector $\boldsymbol{\sigma}(\mathbf{p})$ such that each element takes values in the range $(0,1)$ and the sum of all elements is 1 . That is, the output of the fully connected layer 3 in Fig. 6 is transformed into the input with the probability category that the input image belongs to

$$
\boldsymbol{\sigma}(\mathbf{p})_{i_{1}}=\frac{\mathrm{e}^{p_{i j}}}{\sum_{i_{1}=1}^{N} \mathrm{e}^{p_{i_{1}}}}
$$

among which, $p_{i_{1}}$ is the $i_{1}$-st element of the $N$-dimensional vector $\mathbf{p}$.

To prevent the gradient from exploding or vanishing and falling into local optima, the activation function input of the network layer is standardized by batch normalization to avert the training trapped into the nonlinear saturation region, thus speeding up the convergence of the network. For a certain batch of data $\left\{x_{1}, x_{2}, \ldots, x_{n}\right\}$ in training, the network layer can either be the input or output of the middle layer. The data batch normalization can be expressed as

$$
\begin{aligned}
\mu & =\frac{1}{n} \sum_{i=1}^{n} x_{i} & \sigma^{2} & =\frac{1}{n} \sum_{i=1}^{n}\left(x_{i}-\mu\right) \\
\widehat{x}_{i} & =\frac{x_{i}-\mu}{\sqrt{\sigma^{2}+\varepsilon}} & \widehat{y}_{l} & =\gamma \widehat{x}_{i}+\beta
\end{aligned}
$$

Batch normalization uses optimization to change the magnitude of the data variance and mean value location, where $\gamma$ and $\beta$ are parameters that need to be learned by the network, $\varepsilon$ is the given parameter, and the normalization ends up with $\widehat{y}_{l}$ as the output.

\subsection{Data enhancement}

An effective way to improve the generalization ability of CNN is to perform data augmentation on the training dataset, such as artificially create dummy data and add it to the training set. Four kinds of road image data are collected, and then image processing is carried out on some of the road images, i.e., adjusting the image brightness and darkness, panning the image by a number of pixels in the horizontal or vertical direction, flipping and cropping the image at a small angle, so as to simulate the influence of the external natural environment and vehicle vibration on the image quality during sampling and to achieve the purpose of data enhancement. The original and enhanced images are shown in Fig. 7, and the processing parameters are given in Table 2. 
(a)

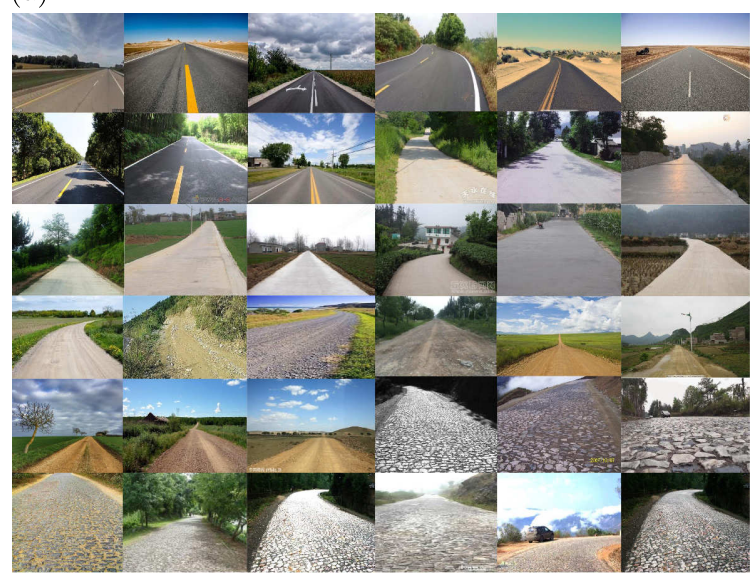

(c)

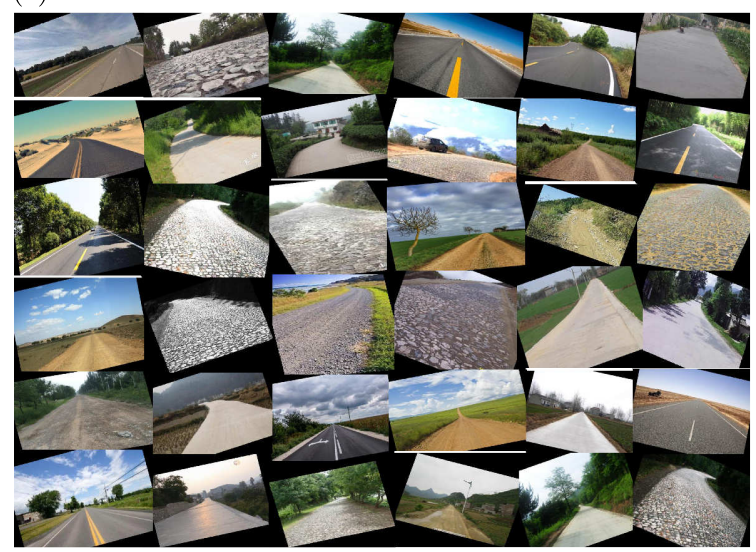

(b)

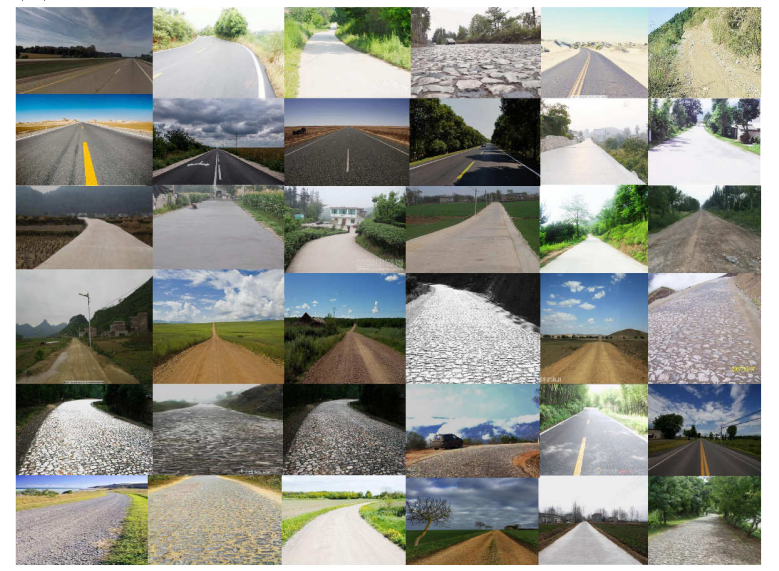

(d)

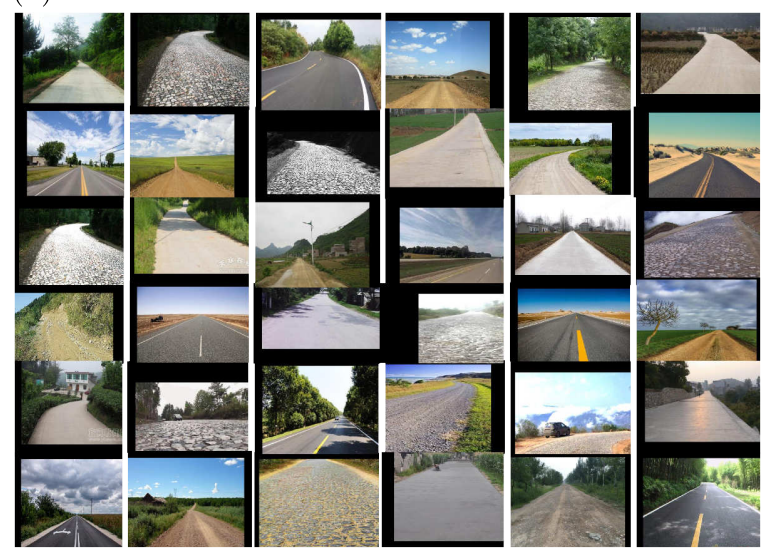

Fig. 7. Original and enhanced road images: (a) original images, (b) brightness adjustment, (c) randomly rotation and cut, (d) randomly shift

Table 2. Image processing parameters

\begin{tabular}{|c|c|c|c|c|}
\hline Processing & Rotation & Cropping & Panning & Brightness \\
\hline \hline Range & $-20-20$ & $-20-20$ & $-40-40$ & $50 \%$ \\
\hline Unit & degree & degree & pixel & - \\
\hline
\end{tabular}

\section{Simulations}

The overall flowchart of the proposed road recognition-based semi-active suspension control method is shown in Fig. 8, which consists of road image collection, image autoencoder, road type recognition and semi-active suspension control. It can be seen from Fig. 8 that the road images are collected by the camera as the input, and then transfered to the pre-trained autoencoder, and the decodered images are recognized by the pre-trained CNN. Based on the recognition results, the semi-active suspension control parameters optimized by the genetic algorithm are selected to achieve the purpose of adaptive control under different road surfaces to improve vehicle performance. Four types of roads are simulated, and the CNN recognition range can be widened by increasing the types of road surfaces in the dataset for common cases.

\subsection{Network training and simulation}

This paper collects the image data of the four types mentioned in Section 2.3, i.e., asphalt road, cement road, gravel road and pebble road. Each road type has 10000 samples after data 


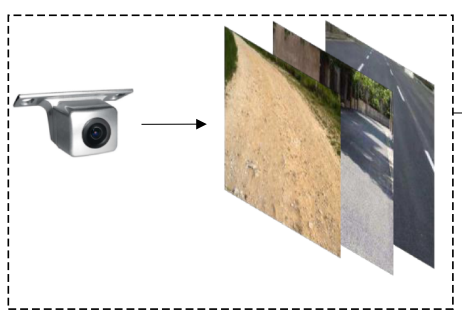

Road image collection

Semi-active suspension control

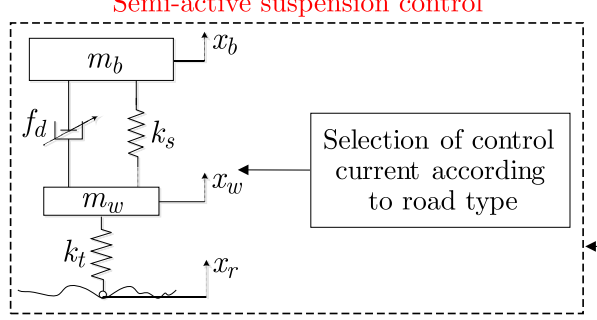

Fig. 8. Road recognition-based suspension control
Road type recognition

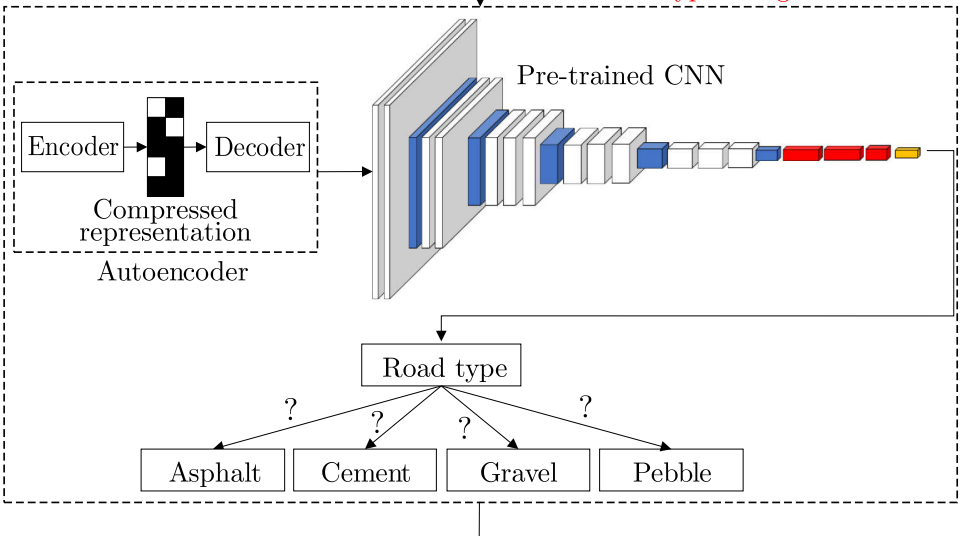

enhancement, for a total of 40000 samples, where 30000 samples are used for training and 10000 samples are used for validation. Each input sample is processed as a $228 \times 228 \times 3$ RGB color image as the input of the autoencoder, and the decoded images are trained through the CNN. The convolution, pooling, error back propagation update weights and other operations of the CNN need to perform a large number of matrix operations, to speed up the network training, the GPU is invoked for network training.

The accuracy and loss function value curves of the CNN training and verification are shown in Fig. 9. It can be seen from the figure that the training process converges after 300 iterations. The numerical accuracy is close to 1 , and the loss function value is close to 0 .
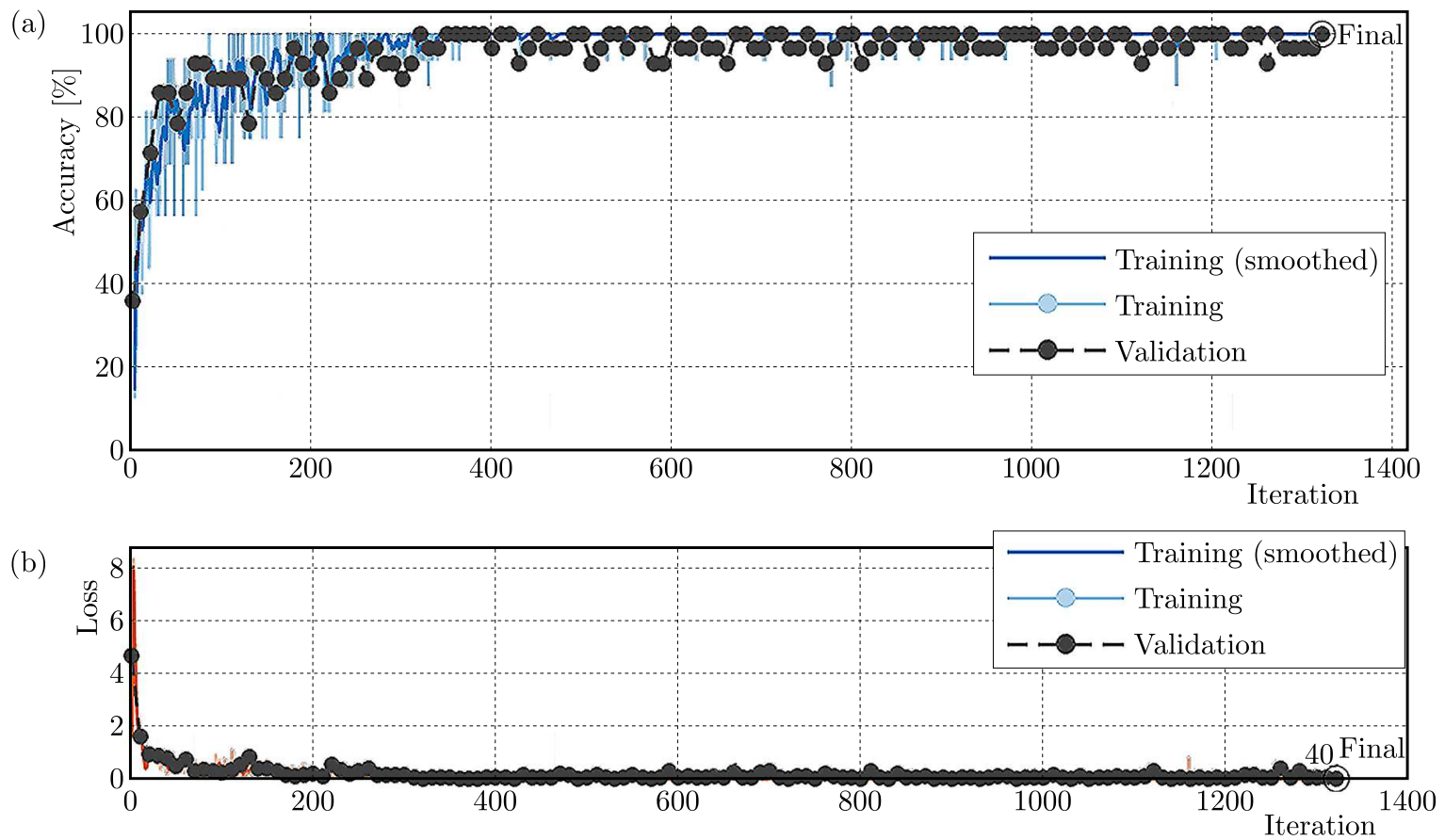

Fig. 9. Training progress: (a) training accuracy, (b) loss function 
After training, 12 samples were randomly selected from the validation set and imported into the trained Network, and the recognition results are shown in Fig. 10. The recognition results and corresponding probabilities are given at the bottom of each sub-picture. According to the figure, the trained Network has good recognition as well as generalization ability for the four typical roads collected in this paper.

(a)

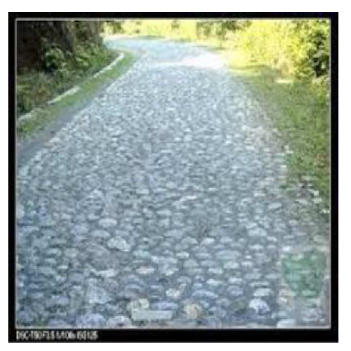

(e)

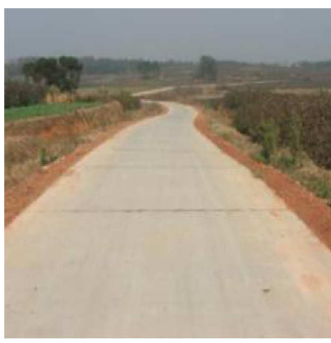

(i)

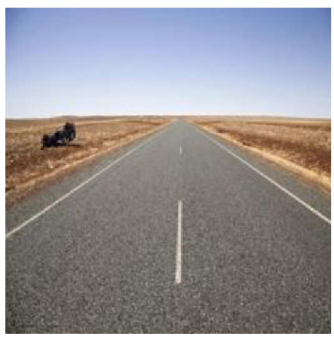

(b)
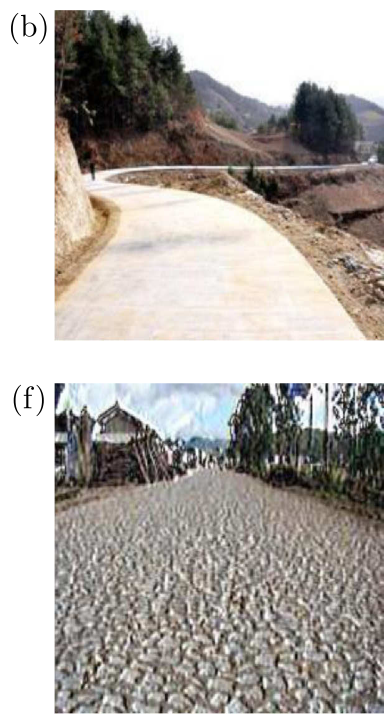

(j)

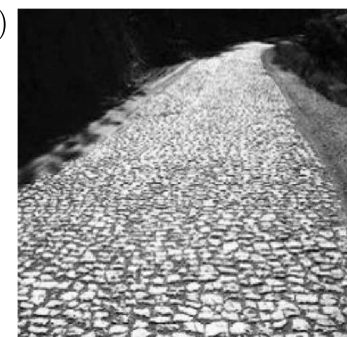

(c)

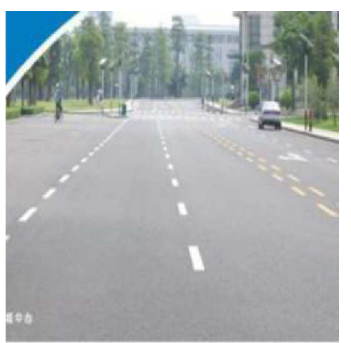

(g)

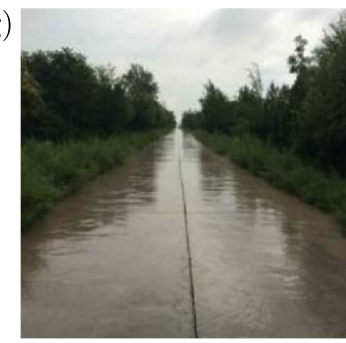

(k)

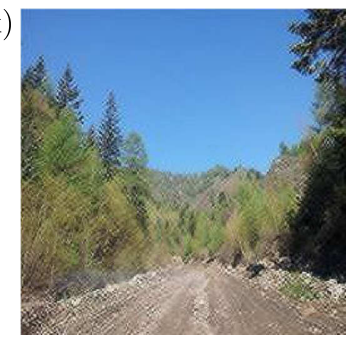

(d)

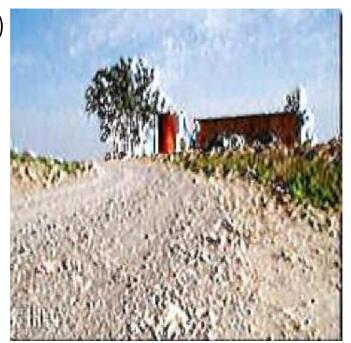

(h)

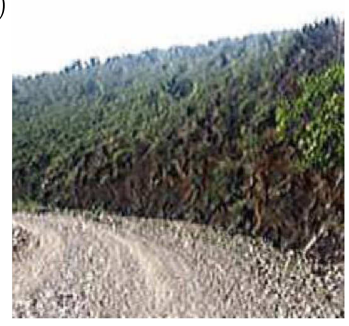

(1)

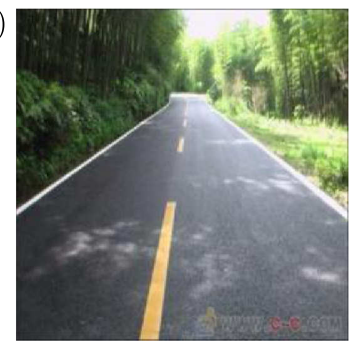

Fig. 10. Random image test results

\subsection{Simulation results of controllable suspension}

To achieve good comfort and handling stability for different types of road profiles, it is necessary to provide different control currents of MR shock absorbers for different road types to obtain suitable damping characteristics. On the basis of the quarter vehicle model, the optimal control current of the MR damper with different road excitations is obtained as the optimization variable, suspension deflection as the constraint, and the root mean square (RMS) value of sprung mass acceleration and tire deflection as the optimization objectives. In this paper, the multiobjective optimization problem is transformed into a sing-objective optimization problem by assigning weight coefficients to the optimization objectives, and then the genetic algorithm is employed to obtain the optimal solution set of the model. The determined objective function is given as follows

$$
\mathrm{obj}=w 1 * \frac{B A_{\text {semt }}}{B A_{\text {pas }}}+w_{2} \frac{D T D_{\text {semt }}}{D T D_{\text {pas }}}
$$

where $B A_{\text {semt }} / B A_{\text {pas }}$ and $D T D_{\text {semt }} / D T D_{\text {pas }}$ are the ratio of the sprung mass acceleration RMS value and the tire deflection RMS value of the passive and semi-active suspension, respectively. $w_{1}$ and $w_{2}$ are the weighting factors of the above two ratios, respectively, and $w_{1}+w_{2}=1$. 
Elite operation is used in the genetic algorithm, 20 outstanding individuals are selected for direct inheritance in each generation without participating in crossover and variation, the population size is 200 , the number of generations is 50 , the crossover and variation probabilities are set to 0.3 and 0.1 , respectively, and the control current is between $0 \mathrm{~A}$ and $2 \mathrm{~A}$. The road profile input adopts the collected time domain road roughness signals. As the riding comfort and hand stability are conflicted with each other, different weighting factors should be assigned to the objective function according to the specific road surface, e.g., vehicle handling stability is considered on poor roads, while riding comfort is the main concern on good roads. The suspension parameters are listed in Table 3. The weighting coefficients of the objective function under different road excitations and the optimized control currents are shown in Table 4.

Table 3. Suspension parameters

\begin{tabular}{|l|c|}
\hline \multicolumn{1}{|c|}{ Parameter } & Value \\
\hline \hline Sprung and unsprung mass & $410 \mathrm{~kg} / 40 \mathrm{~kg}$ \\
\hline Suspension stiffness & $2000 \mathrm{~N} / \mathrm{m}$ \\
\hline Damping coefficient & $1700 \mathrm{Ns} / \mathrm{m}$ \\
\hline Tire stiffness & $185000 \mathrm{~N} / \mathrm{m}$ \\
\hline
\end{tabular}

Table 4. Weighting coefficients and optimized control current

\begin{tabular}{|l|c|c|c|}
\hline Road type & $w_{1}$ & $w_{2}$ & Control current $[\mathrm{A}]$ \\
\hline \hline Asphalt & 0.7 & 0.3 & 0.0489 \\
\hline Cement & 0.6 & 0.4 & 0.0887 \\
\hline Gravel & 0.3 & 0.7 & 0.1976 \\
\hline Pebble & 0.2 & 0.8 & 0.3094 \\
\hline
\end{tabular}

The time domain response of sprung mass acceleration, suspension deflection and tire deflection are plotted in Figs. 11a-11c. From the figures, it can be found that the sprung mass accelerations are effectively improved with the controllable suspension for asphalt and cement roads with good driving conditions, and the suspension deflections and tire deflections are similar to that of the passive system. On the contrary, when driving on gravel and pebble roads, the sprung mass accelerations of the controllable suspension degraded compared to the passive suspension, but the tire deflections are reduced, which effectively improves handling stability of vehicles on poor road surfaces.

The PSD comparison of the sprung mass acceleration and tire deflection with different road excitations are given in Figs. 12a-12d. As shown in Figs. 12a and 12b, it can be observed that compared with the passive suspension system, the controlled system considerably reduces the sprung mass vibration in the natural frequency range between $1 \mathrm{~Hz}$ and $2 \mathrm{~Hz}$ with the excitation of asphalt and cement roads, and the tire deflection amplitudes have increased in the range of $10 \mathrm{~Hz}$ to $15 \mathrm{~Hz}$. The frequency response comparisons with gravel and pebble road excitations are plotted in Figs. 12c and 12d, from where it can be seen that the tire deflections have a lower magnitude in high frequencies compared with the passive system, and there is a slight deterioration observed in the sprung mass vibrations. The simulation results indicate that the system responses of the controllable suspension are consistent with the weighting coefficients selection on different road types. 

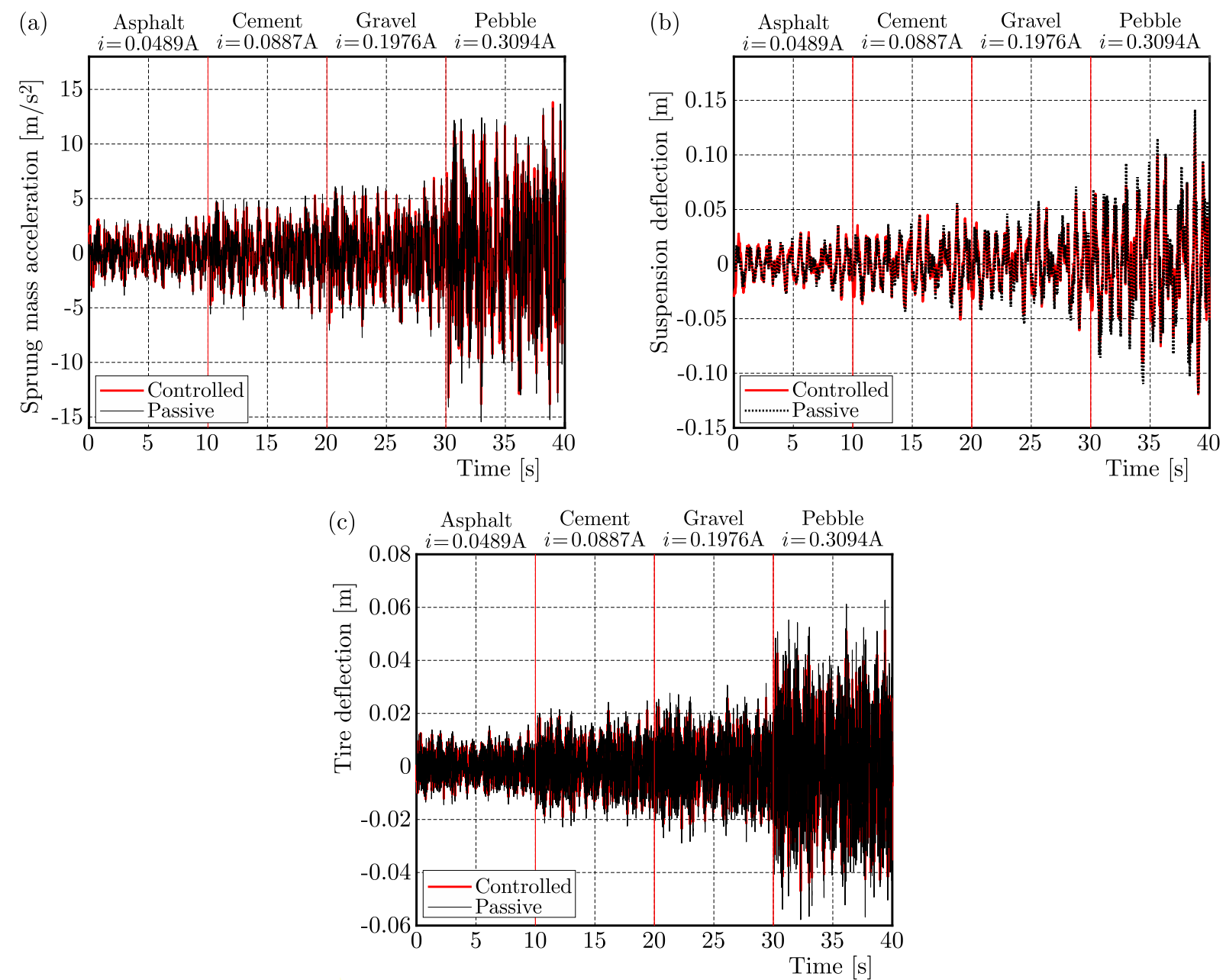

Fig. 11. Suspension performance under different road profiles: (a) vertical acceleration, (b) suspension deflection, (c) tire deflection

\section{Conclusions}

The study presents an adaptive controllable suspension control strategy based on road recognition. A parametric MR damper based semi-active suspension model is established, and information of four typical roads is collected. To avoid a complex pre-process and improve classification efficiency, an autoencoder based CNN framework is applied to compress road images and extract the main features of the road surface. The network architecture is composed of an autoencoder network and multi-layer neural networks, and the parameters of CNNs are well trained by the pre-training progress. With the road recognition results, the choice of the control current for different road types is then transformed into a multi-object optimization problem, and a genetic algorithm is utilized to solve the problem. The simulation results reveal that the proposed road estimation method can accurately identify the road type, and based on the recognition results, the control strategy can adaptively adjust the control signals to suit different road surfaces.

\section{Acknowledgments}

The present research was supported by the Natural Science Foundation of Shannxi Province (No. 2021JQ-857), Shaanxi Provincial Key Research and Development Project (No. 2020ZDLGY16-01), "Transportation Vehicle Inspection, Diagnosis and Maintenance Technology" Key Laboratory Project of Transportation Industry (No. JTZL2002) and Scientific Research Project of Education Department of Shaanxi Provincial (No. 21JK0700). 
(a)
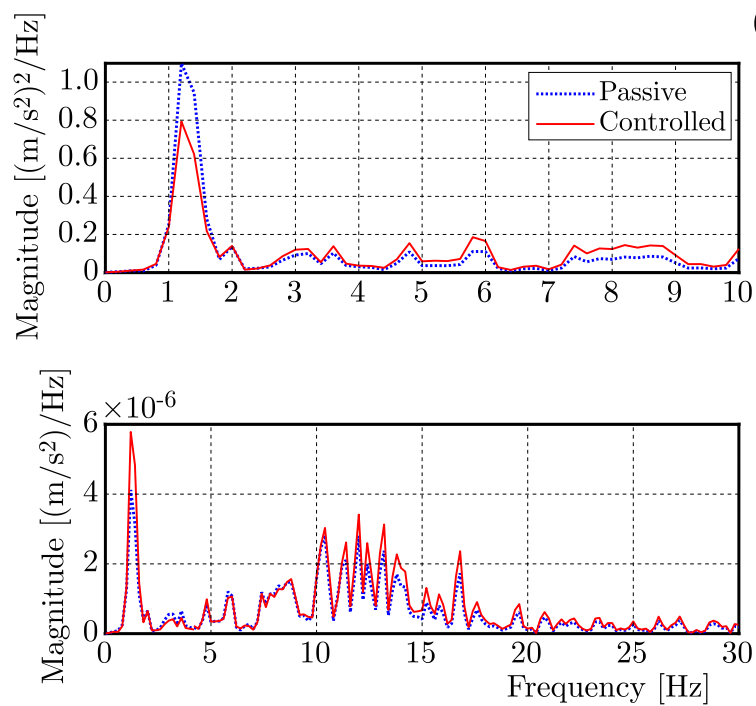

(c)
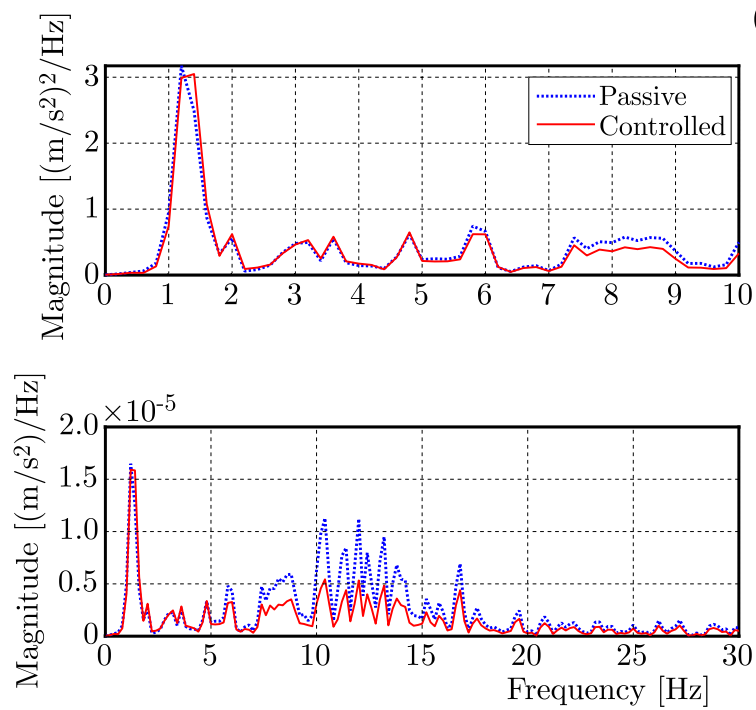

(b)
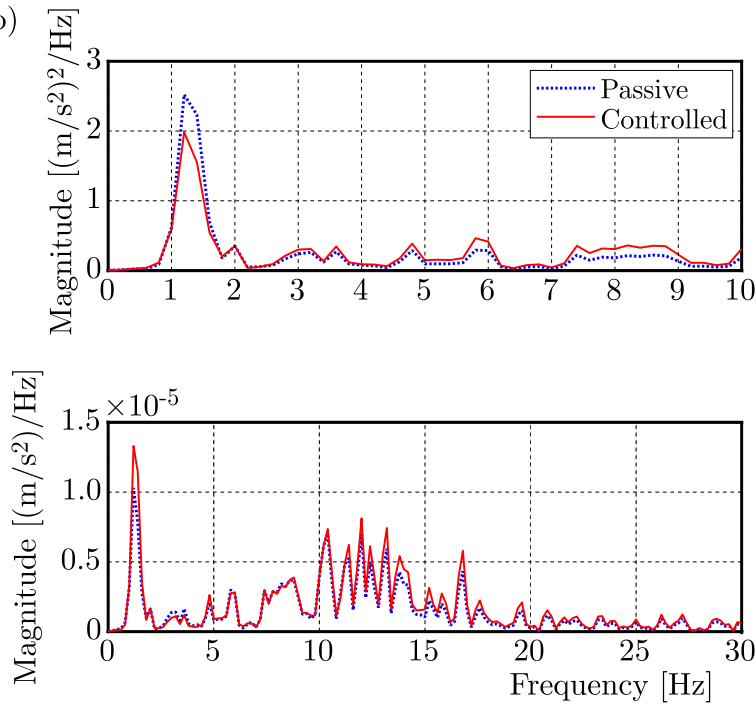

(d)
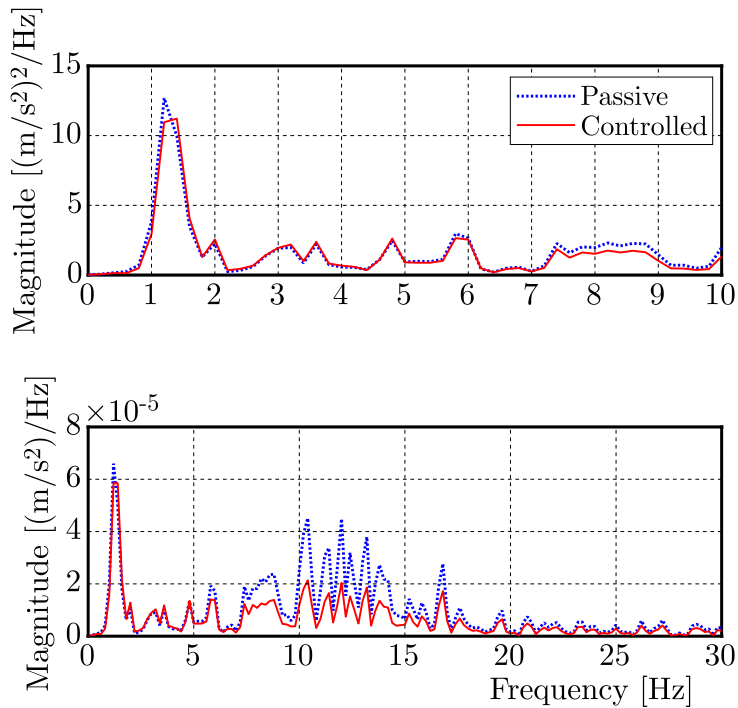

Fig. 12. PSD comparison of different road excitations: (a) asphalt road, (b) cement road, (c) gravel road, (d) pebble road

\section{References}

1. Beкhti M.A., Kobayashi Y., 2015, Prediction of vibrations as a measure of terrain travers ability in outdoor structured and natural environments, Proceedings of the rth Pacific-Rim Symposium on Image and Video Technology, Auckland, New Zealand: Springer, 282-294

2. Doumiati M., Victorino A., Charara A., Lechner D., 2011, Estimation of road profile for vehicle dynamics motion: experimental validation, Proceedings of the 2011 American Control Conference, San Francisco, CA, USA, 5237-5242

3. Fauriat W., Mattrand C., Gayton N., Beakou A., Cembrzynski T., 2016, Estimation of road profile variability from measured vehicle responses, Vehicle System Dynamics, 54, 5, 585-605

4. Gong, M., Chen, H., 2020, Variable damping control strategy of a semi-active suspension based on the actuator motion state, Journal of Low Frequency Noise, Vibration and Active Control, 39, $3,787-802$

5. Hinton G.E., Zemel R.S., 1994, Autoencoders, minimum description length, and Helmholtz free energy, Advances in Neural Information Processing Systems, 6, 3-10 
6. KrzyŻyński T., Maciejewski I., 2019, Computational method for shaping the vibro-isolation properties of semi-active and active systems, Archives of Mechanics, 71, 4-5, 291-313

7. Kwok N.M., Ha Q.P., Nguyen Thi H., Li J., Samali B., 2006, A novel hysteretic model for magnetorheological fluid dampers and parameter identification using particle swarm optimization, Sensors and Actuators A. Physical, 132, 2, 441-451

8. Li Z., Kalabic U.V., Kolmanovsky I.V., Atkins E., Lu J., Filev D., 2016, Simultaneous road profile estimation and anomaly detection with an input observer and a jump diffusion process estimator, 2016 American Control Conference (ACC), IEEE, 1693-1698

9. Liu W., Wang R., Ding R., Meng X., Yang L., 2020, On-line estimation of road profile in semiactive suspension based on unsprung mass acceleration, Mechanical Systems and Signal Processing, 135, 1, 106370

10. Maciejewski I., Krzyżyński T., Pecolt S., Chamera S., 2019, Semi-active vibration control of horizontal seat suspension by using magneto-rheological damper, Journal of Theoretical and Applied Mechanics, 57, 2,411-420

11. Morales A.L., Nieto A.J., Chicharro J.M., Pintado P., 2016, A semi-active vehicle suspension based on pneumatic springs and magnetorheological dampers, Journal of Vibration and Control, 24, 4, 808-821

12. Peterson K., Ziglar J., Rybski P.E., 2008, Fast feature detection and stochastic parameter estimation of road shape using multiple lidar, 2008 IEEE/RSJ International Conference on Intelligent Robots and Systems, 612-619

13. Qin Y., He C., Shao X., Du H., Xiang C., Dong M., 2018, Vibration mitigation for in-wheel switched reluctance motor driven electric vehicle with dynamic vibration absorbing structures, Journal of Sound and Vibration, 419, 249-267

14. Qin Y., Langari R., Wang Z., Xiang C., Dong M., 2017, Road excitation classification for semi-active suspension system with deep neural networks, Journal of Intelligent and Fuzzy Systems, 33, 3, 1907-1918

15. Qin Y., Reza L., Gu L., 2014, The use of vehicle dynamic response to estimate road profile input in time domain, ASME Dynamic System Control Conference (DSCC), San Antonio, TX, USA, DOI: 10.1115/DSCC2014-5978

16. Qin Y., Wang Z., Xiang C., Hashemi E., Khajepour A., Huang Y., 2019, Speed independent road classification strategy based on vehicle response: Theory and experimental validation, Mechanical Systems and Signal Processing, 117, 653-666

17. Qin Y., Wei C., TANG X., Zhang N., 2019, A novel nonlinear road profile classification approach for controllable suspension system: Simulation and experimental validation, Mechanical Systems and Signal Processing, 125, 6, 79-98

18. Rath J.J., Veluvolu K.C., Defoort M., 2015, Simultaneous estimation of road profile and tire road friction for automotive vehicle, IEEE Transactions on Vehicular Technology, 64, 10, 4461-4471

19. Sun J., Cong J.Y., Gu L., Dong M., 2019, Higher order sliding mode control for active suspension systems subject to actuator faults and disturbances, Proceedings of the Institution of Mechanical Engineers Part K-Journal of Multi-Body Dynamics, 233, 2, 280-298

20. Vitkari V.V., Varpula T., Kantanen M., 2009, Road-condition recognition using 24-GHz automotive radar, IEEE Transactions on Intelligent Transportation Systems, 10, 4, 639-648

21. Zhang Z., Deng F., Huang Y., Bridgelall R., 2015, Road roughness evaluation using inpavement strain sensors, Smart Materials and Structures, 24, 11, 115029 